\title{
Palaeolithic diet ("stone age" diet)
}

\author{
Staffan Lindeberg
}

Department of Clinical Sciences, Lund University, Lund, Sweden

Abstract

A popular diet in Sweden today is the palaeolithic diet, where lean meat, fish, vegetables, fruit, roots and nuts are dietary staples, while cereals, dairy products, salt and processed fat and sugar are avoided. The underlying rationale is that foods that were available during the evolution of primates, up to the emergence of fully modern humans, are healthier than recently introduced ones (dairy products, cereals, beans, refined fat, sugar, etc.), since our digestive and metabolic systems were not designed for the latter group of foods. Variation in plant foods, another principle based on evolutionary biology, is recommended to avoid high intakes of potentially harmful bioactive substances. It is not known whether palaeolithic diets are more, or less, effective than other diets in weight reduction.

Keywords: evolutionary medicine; metabolic syndrome; overweight; popular diets; Western disease

\section{Introduction}

The Palaeolithic, the old stone age, started around 2.5 million years BP, when humans first made use of stone tools. The period ended with the emergence of agriculture approximately 10000 years ago (5000 years ago in Scandinavia). During the Palaeolithic, wild meat, fish, insects, eggs, fruit, berries, vegetables and nuts were consumed in varying proportions depending on the ecological niche $(1,2)$. Seeds and beans were rarely eaten and never in large amounts on a daily basis. Dairy products and refined fat and sugar were not available.

A large variety of plant species was consumed, as inferred from surveys in contemporary huntergatherers (3) and free-living primates (4). Accordingly, the intake of any single plant-derived bioactive substance (e.g. terpenoids, alkaloids, glucosinolates, tannins, phytoestrogens and lectins) was low. Many, if not most, of these bioactive substances are considered to be part of the defence system against herbivory: plants do not "want" to be eaten $(5,6)$. The highest concentration of bioactive substances is typically found in seeds and beans.

\section{Palaeolithic lifestyles may prevent Western disease}

Overweight and the metabolic syndrome have been conspicuously absent in twentieth century populations with palaeolithic lifestyles, as shown in several surveys (7). Cardiovascular disease including stroke was apparently unknown in East Africa before the transition to a modern lifestyle. One such population was studied in Kitava, Trobriand Islands, Papua New Guinea (8-13). In Kitava, food was constantly available in excess, suggesting that food shortage is an overestimated cause of leanness in palaeolithic populations. The level of physical activity of Kitavans was roughly estimated at 1.7 multiples of the basal metabolic rate, which is the same as in Western males who are moderately active at work and during leisure activities.

Ageing in the Western world is very different to that in palaeolithic populations. If, at the age of 50 years, Swedish men and women had the body mass index of Kitavans, they would weigh 19 and $22 \mathrm{~kg}$, respectively, less than they actually do (9). These striking differences indicate that typical ageing in the modern world is biologically abnormal. Advanced atherosclerosis is seen in virtually every elderly Westerner, irrespective of body mass index (14). One of the main suspects is diet.

Observational studies cannot prove causal relationships, but they lend support to the notion that a palaeolithic diet may prevent overweight and Western disease, even when food is available in excess.

\section{Age structure and genetics may not be the most important explanations}

Palaeolithic populations do not lack elderly individuals, although the high mortality in infancy and childhood dramatically lowers average life expectancy. Among hunter-gatherers, estimated life 
expectancy at birth may be below 40 years, while life expectancy at age 50 years may be close to European levels of today (15). Accordingly, there is no obvious reason to suspect that a different age structure is the main explanation for the apparent absence of cardiovascular disease in non-Western populations.

The role of genetics is often misinterpreted, as if genes could work independently of the environment. Although familial heritage apparently is a strong determinant within Westernized populations, genetic factors do not appear to explain the rarity of overweight, cardiovascular disease and diabetes among traditional populations. On the contrary, after adopting a Western lifestyle non-Europeans seem more prone to developing these disorders compared with populations of northern European ancestry (16-19).

\section{Nutritional characteristics of palaeolithic diets}

A diet based on lean meat, fish, vegetables, roots, fruit and nuts can easily meet human nutritional requirements according to the Nordic Nutrition Recommendations, except that calcium intake often is too low, especially when the intake of green leafy vegetables is limited. Adding milk to a palaeolithic diet thus increases calcium intake but does not necessarily prevent osteoporosis $(1,20)$. There are no other well-founded benefits of adding dairy products to a palaeolithic diet. The addition of wholegrain cereals or beans provides no known benefit, but may increase the risk of vitamin and mineral deficiencies.

\section{This is not a low-carb diet}

A palaeolithic diet is often high in protein, typically $15-35 \%$ of energy $(E \%)$, but not necessarily low in carbohydrate (3). It should not be confused with the Atkins diet or similar programmes based on the assumption that a high intake of carbohydrates promotes insulin resistance and dyslipidaemia. Our primate ancestors are considered to have been specialized fruit-eaters for more than 40 million years (21), and it is unlikely that our metabolism has lost the capacity to handle high amounts of carbohydrates in the relatively short periods as palaeolithic hunters. In Kitava, carbohydrates provided nearly $70 \mathrm{E} \%$, but overweight was apparently absent and insulin sensitivity was markedly higher than in a randomly selected Swedish population $(12,22)$.
Palaeolithic diets do not consist of meat in exchange for vegetables: both are included. The proportion of animal products (meat, fish, shellfish, insects) as opposed to plant foods varied dramatically among our human ancestors depending on habitat and period. There is no good evidence to suggest that lean, whole meat promotes overweight or related diseases.

\section{Caloric restriction without food restriction}

There is uncertainty regarding the health benefits of weight loss. Several prospective observational cohort studies suggest increased mortality in moderately overweight subjects who voluntarily lose weight, compared with those who are weight stable (23). Losing the same amount of weight may hypothetically have different long-term health effects depending on the weight-reduction programme. For example, restricting the amount of food without improving the quality may increase the risk of suboptimal intake of micronutrients. Relevant dietary intervention trials with hard clinical endpoints (mortality, myocardial infarction, etc.) are lacking.

Animal experiments suggest that caloric restriction has several positive health effects (24). Intuitively, lean meat, fish, vegetables and fruit may be prudent choices in an energy-restricted diet model. Indirect evidence suggests that such foods are satiating owing to their low energy density, but this has not been properly tested in clinical trials.

\section{Conclusion}

The palaeolithic diet is not primarily a weight-loss programme. Rather, it is part of a theoretical template for health promotion $(1,25)$. It has not been proven to prolong life, and the same is true for low-fat, high-fibre diets. Overweight subjects who wish to eat a palaeolithic diet can do so without obvious risks. However, calcium supplementation may be considered.

\section{References}

1. Lindeberg S. Maten och folksjukdomarna - ett evolutionsmedicinskt perspektiv. Lund: Studentlitteratur; 2003.

2. Lindeberg S, Cordain L, Eaton SB. Biological and clinical potential of a palaeolithic diet. J Nutr Environ Med 2003; 13: 1-12.

3. Cordain L, Miller JB, Eaton SB, Mann N, Holt SH, Speth JD. Plant-animal subsistence ratios and macro- 
nutrient energy estimations in worldwide hunter-gatherer diets. Am J Clin Nutr 2000; 71: 682-92.

4. Basabose AK. Diet composition of chimpanzees inhabiting the Montane forest of Kahuzi, Democratic Republic of Congo. Am J Primatol 2002; 58: 1-21.

5. Herrera CM, Pellmyr O. eds. Plant-animal interactions. An evolutionary approach. Oxford: Blackwell; 2002.

6. Wynne-Edwards KE. Evolutionary biology of plant defenses against herbivory and their predictive implications for endocrine disruptor susceptibility in vertebrates. Environ Health Perspect 2001; 109: 443-8.

7. Lindeberg S. Apparent absence of cerebrocardiovascular disease in Melanesians. Risk factors and nutritional considerations - the Kitava Study. Lund: University of Lund; 1994.

8. Lindeberg S, Lundh B. Apparent absence of stroke and ischaemic heart disease in a traditional Melanesian island: a clinical study in Kitava. J Intern Med 1993; 233: $269-75$.

9. Lindeberg S, Nilsson-Ehle P, Terént A, Vessby B, Scherstén B. Cardiovascular risk factors in a Melanesian population apparently free from stroke and ischaemic heart disease - the Kitava study. J Intern Med 1994; 236: $331-40$.

10. Lindeberg S. Cardiovascular disease in the tropics, ethics, and need for action (Letter). Lancet 1995; 345: 384.

11. Lindeberg S, Berntorp E, Nilsson-Ehle P, Terent A, Vessby B. Age relations of cardiovascular risk factors in a traditional Melanesian society: the Kitava Study. Am J Clin Nutr 1997; 66: 845-52.

12. Lindeberg S, Eliasson M, Lindahl B, Ahrén B. Low serum insulin in traditional Pacific Islanders - the Kitava Study. Metabolism 1999; 48: 1216-9.

13. Lindeberg S. Stroke in Papua New Guinea. Lancet Neurol 2003; ii: 273.

14. Stary HC. Lipid and macrophage accumulations in arteries of children and the development of atherosclerosis. Am J Clin Nutr 2000; 72: 1297-306S.

15. Pennington R. Hunter-gatherer demography. In: Panter-Brick C, Layton RH, Rowley-Conwy P, eds. Hunter-gatherers. An interdisciplinary perspective. Cambridge: Cambridge University Press; 2001. p. 170204.
16. King H. The epidemiology of diabetes mellitus in Papua New Guinea and the Pacific: adverse consequences of natural selection in the face of sociocultural change. In: Attenborough RD, Alpers MP, eds. Human biology in Papua New Guinea. The small cosmos. Oxford: Clarendon Press; 1992. p. 363-72.

17. Zimmet P. Epidemiology of diabetes and its macrovascular manifestations in Pacific populations: the medical effects of social progress. Diabetes Care 1979; 2: 144-53.

18. Brand Miller JC, Colagiuri S. The carnivore connection: dietary carbohydrate in the evolution of NIDDM. Diabetologia 1994; 37: 1280-6.

19. Allen JS, Cheer SM. The non-thrifty genotype. Curr Anthropol 1996; 37: 831-42.

20. Michaelsson K, Melhus H, Bellocco R, Wolk A. Dietary calcium and vitamin $\mathrm{D}$ intake in relation to osteoporotic fracture risk. Bone 2003; 32: 694-703.

21. Dudley R. Fermenting fruit and the historical ecology of ethanol ingestion: is alcoholism in modern humans an evolutionary hangover? Addiction 2002; 97: 381-8.

22. Lindeberg S, Söderberg S, Ahren B, Olsson T. Large differences in serum leptin levels between nonwesternized and westernized populations: the Kitava study. J Intern Med 2001; 249: 553-8.

23. Nilsson PM, Nilsson JA, Hedblad B, Berglund G, Lindgärde $F$. The enigma of increased non-cancer mortality after weight loss in healthy men who are overweight or obese. J Intern Med 2002; 252: 70-8.

24. Heilbronn LK, Ravussin E. Calorie restriction and aging: review of the literature and implications for studies in humans. Am J Clin Nutr 2003; 78: 361-9.

25. Cordain L, Eaton SB, Sebastian A, Mann N, Lindeberg $\mathrm{S}$, Watkins BA, et al. Origins and evolution of the Western diet: health implications for the 21 st century. Am J Clin Nutr 2005; 81: 341-54.

\section{Staffan Lindeberg}

Department of Clinical Sciences

Lund University

SE-227 38 Lund

Sweden

Email: staffan.lindeberg@med.lu.se 